\title{
WHICH INTEGERS ARE REPRESENTABLE AS THE PRODUCT OF THE SUM OF THREE INTEGERS WITH THE SUM OF THEIR RECIPROCALS?
}

\author{
ANDREW BREMNER, RICHARD K. GUY, AND RICHARD J. NOWAKOWSKI \\ In memory of Dick Lehmer, with thanks for the Western Number Theory conferences, and for \\ launching at least one of us on a career in number theory.
}

\begin{abstract}
For example, the integer 564 is so representable by the three integers

122442005010002877811635117117995213613513491867 ,

-3460695868425504865645892262188752089713065424460 and

74807191015302527837945836017146464948205905528060 .
\end{abstract}

\section{INTRODUCTION}

Melvyn J. Knight has asked which integers $n$ can be represented as

$$
n=(x+y+z)\left(\frac{1}{x}+\frac{1}{y}+\frac{1}{z}\right)
$$

where $x, y, z$ are integers, perhaps positive ones. Rewrite (1) as

$$
(x+y+z)(y z+z x+x y)=n x y z .
$$

If $(x, y, z)$ is a solution, so is any permutation. So is $(k x, k y, k z)$ for any $k$. By putting $k=-1$ we see that at most one of $x, y, z$ need be negative. Also, it suffices to find rational solutions, and to retrieve primitive integer solutions, with $\operatorname{gcd}(x, y, z)=1$, by an appropriate choice of rational $k$. If $(x, y, z)$ is a solution, so is $(1 / x, 1 / y, 1 / z)$. Indeed, (2) is quadratic in $x, y$ and $z$, and solutions occur in reciprocal pairs:

$$
\begin{aligned}
& n \begin{array}{ccccccccc}
-10 & -4 & 0 & 1 & 9 & 10 & 11 & 14 & 15
\end{array} \\
& (-12,3,1)(-6,2,1)(-2,1,1) \quad(-1,1 \text {, any })(1,1,1)(2,2,1)(6,3,2) \quad(10,3,2) \quad(6,3,1)
\end{aligned}
$$

The right side of (1) may be written as

$$
9+\frac{(y-z)^{2}}{y z}+\frac{(z-x)^{2}}{z x}+\frac{(x-y)^{2}}{x y},
$$

Received by the editor May 19, 1992.

1991 Mathematics Subject Classification. Primary 11D25, 11G05, 11Y50. 
so that if $x, y, z$ are all positive, no integer less than 9 can be represented, and 9 only with $x=y=z$.

\section{A FAMILY OF ELLIPTIC CURVES}

Write (2) as

$$
x^{2}(y+z)+x\left(y^{2}+(3-n) y z+z^{2}\right)+y z(y+z)=0,
$$

so that

$$
x=\frac{-y^{2}+(n-3) y z-z^{2}-\Delta}{2(y+z)},
$$

where

$$
\Delta^{2}=y^{4}-2(n-1) y^{3} z+\left(n^{2}-6 n+3\right) y^{2} z^{2}-2(n-1) y z^{3}+z^{4} .
$$

By standard arguments (see, e.g., Mordell [4, p. 77-78]), (5) is birationally equivalent to the elliptic curve

$$
\tau^{2}=\sigma\left(\sigma^{2}+\left(n^{2}-6 n-3\right) \sigma+16 n\right),
$$

and this allows us to write down maps between (3) and (6) as follows:

$$
\sigma=-4(y z+z x+x y) / z^{2}, \quad \tau=2(\sigma-4 n) y / z-(n-1) \sigma
$$

and

$$
\frac{x, y}{z}=\frac{ \pm \tau-(n-1) \sigma}{2(4 n-\sigma)} .
$$

The solutions to Knight's problem for any particular value of $n$, if there are any, will correspond to rational points on (6). By the Mordell-Weil theorem these form a group comprising a finite torsion group and a finitely generated infinite group with $r$ generators, where $r$ defines the rank of the curve. Geometrically, the curve (6) has two components, an "egg" for values $\sigma<0$, and an infinite branch when $\sigma>0$.

We characterize representations in which $x, y, z$ are all positive. From (7), $\sigma<0$. If $n<0$, then, from (1) or (2), at least one of $x, y, z$ must be negative. If $n>0$, then, from (8), $x, y, z$ will all be positive just if $(\sigma<0$ and) $(n-1)^{2} \sigma^{2}>\tau^{2}$, i.e.,

$$
(n-1)^{2} \sigma^{2}>\sigma\left(\sigma^{2}+\left(n^{2}-6 n-3\right) \sigma+16 n\right) .
$$

Divide by $\sigma \quad(<0)$ :

$$
\begin{gathered}
(n-1)^{2} \sigma<\sigma^{2}+\left(n^{2}-6 n-3\right) \sigma+16 n, \\
(\sigma-(2 n+2))^{2}>(2 n-2)^{2},
\end{gathered}
$$

which is always true if $n>0$ and $\sigma<0$.

Necessary and sufficient conditions for a positive representation are $n>0, \sigma<0$.

If there is a rational point on the egg, then there are such points on the infinite branch; i.e., if there is a representation with positive integers, then there will also be representations with $x, y, z$ not all of the same sign. But the converse 
is not true. It is possible to have an infinite number of rational points on the infinite branch and none on the egg. The smallest such example is $n=29$, which has $(x, y, z)=(5,-26,195)$ and an infinity of other solutions, but none of them positive.

If $P$ is on the egg, then just the odd multiples of $P$ give positive solutions. E.g., for $n=11$ the point $P(-11,55)$ corresponds to the solution $(x, y, z)=$ $(3,1,2)$. The point $2 P\left(\frac{1}{4}, \frac{55}{8}\right)$ gives the nonpositive solution $(7,-5,140)$, while $3 P$ gives $(22243,8177,8874)$. The next smallest positive solution is given by $5 P$ :

$$
\text { (596917970819, 264668812593, 765759090202). }
$$

The only values of $n$ less than 1000 with positive representations are precisely those shown in bold type in Table 1 in $\S 8$. The integer $n=997$ has no positive representation even though it corresponds to a curve of rank three (the solutions $(30,-8455,38418),(390,-1219,572010)$ and $(85,-187068,271740)$ are independent).

\section{Some SINGUlar CASES}

The discriminant of $(6)$ is

$$
\left(n^{2}-6 n-3\right)^{2}-64 n=(n-1)^{3}(n-9),
$$

and it follows that (6) is singular only for $n=0,1$ and 9 .

$n=0: \tau^{2}=\sigma^{2}(\sigma-3), \sigma-3=\mu^{2}$, where $\mu$ is rational, $(\sigma, \tau)=$ $\left(\mu^{2}+3, \pm \mu\left(\mu^{2}+3\right)\right)$ and equation (8) gives

$$
\begin{gathered}
\frac{x, y}{z}=\frac{ \pm \mu\left(\mu^{2}+3\right)+\mu^{2}+3}{-2\left(\mu^{2}+3\right)}=\frac{1 \pm \mu}{-2}, \\
x: y: z=1+\mu: 1-\mu:-2 .
\end{gathered}
$$

For example, any three integers whose sum is zero.

$$
\begin{gathered}
n=1: \tau^{2}=\sigma(\sigma-4)^{2},(\sigma, \tau)=\left(\mu^{2}, \pm \mu\left(\mu^{2}-4\right)\right), \\
\frac{x, y}{z}=\frac{\mp \mu}{2} \\
x: y: z=-\mu: \mu: 2 .
\end{gathered}
$$

For example, any three integers of which two sum to zero.

$$
\begin{gathered}
n=9: \tau^{2}=\sigma(\sigma+12)^{2},(\sigma, \tau)=\left(4 \mu^{2}, \pm 8 \mu\left(\mu^{2}+3\right)\right), \\
\frac{x, y}{z}=\frac{ \pm 8 \mu\left(\mu^{2}+3\right)-32 \mu^{2}}{2\left(36-4 \mu^{2}\right)}=\frac{-\mu(\mu-1)(\mu-3), \mu(\mu+1)(\mu+3)}{\mu^{2}-9}, \\
x: y: z=-\mu(\mu-1)(\mu-3): \mu(\mu+1)(\mu+3): \mu^{2}-9 .
\end{gathered}
$$

Rational values of $\mu$ give integer solutions, with at least one of $x, y, z$ negative. For example, $(2,30,-5)$ and $(15,1,-6)$ are given by $\mu= \pm 2, \pm 5, \pm \frac{3}{2}$, $\pm 9, \pm \frac{1}{3}, \pm \frac{3}{5}$. But to get the obvious (and unique positive) solution $x=y=z$ we have to put $\mu= \pm i \sqrt{3}$.

\section{THE TORSION GROUP}

We assume that the curve is nonsingular, i.e., $n \neq 0,1$ or 9 . The point $(0,0)$ is always of order two. When $n<0$, this point is on the egg, and when 
$n>0$, it is on the infinite branch. There can be other rational points of order two only when (9) is a perfect square, i.e., for the three excluded values, and for $n=10$. The points of inflexion, $(4, \pm 4(n-1))$, are rational and of order three. The points $(4 n, \pm 4 n(n-1))$ are of order six. In the general case the tangents to the curve from the sole point of order two are not rational, so that there are no rational points of order four. The tangents to the curve from the point of inflexion $(4,4 n-4)$ have slopes $\mu$ given by $(\mu+2)^{2}=(n-1)^{2}$ and $(\mu-2)^{2}=(n-1)(n-9)$. The first pair are those of the tangent at the point of inflexion itself, with slope $n-3$, and the tangent at the point $(4 n,-4 n(n-1))$ of order six, with slope $-(n+1)$. The other two slopes are rational only in the excluded cases, and for $n=10$. In all cases except $n=10$, therefore, the torsion group is $\mathbb{Z} / 6 \mathbb{Z}$.

$n=10: \tau^{2}=\sigma(\sigma+5)(\sigma+32)$ displays four more points of order six, namely $(-8, \pm 24)$ and $(-20, \pm 60)$, and the torsion group is isomorphic to $\mathbb{Z} / 2 \mathbb{Z} \times \mathbb{Z} / 6 \mathbb{Z}$. The solutions $(2,1,2)$ and $(2,1,1)$ of $(1)$ given by these additional torsion points are essentially unique since in fact this curve has rank zero and accordingly has no rational points of infinite order. Only for $n=10$ do we obtain a rank-zero curve affording solutions to (1).

The relationships between the permutations and reciprocals of a solution $(x, y, z)$ and the points on the curve (6) are exhibited in the following table, where $\bar{x}=\operatorname{lcm}(x, y, z) / x$, etc.

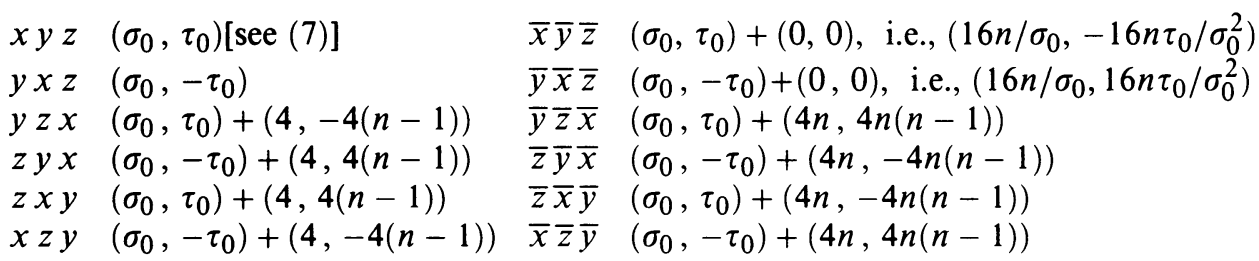

\section{Calculating the Rank}

To find the rank of (6), we first note (see, e.g., Silverman [5, p. 74]) that there are isogenies of degree two between (6) and

$$
T^{2}=S\left(S^{2}-2\left(n^{2}-6 n-3\right) S+(n-1)^{3}(n-9)\right) .
$$

The isogenies are

$$
(S, T)=\left(\frac{\tau^{2}}{\sigma^{2}},\left(1-\frac{16 n}{\sigma^{2}}\right) \tau\right)
$$

and

$$
(\sigma, \tau)=\left(\frac{T^{2}}{4 S^{2}},\left(1-\frac{(n-1)^{3}(n-9)}{S^{2}}\right) \frac{T}{8}\right) .
$$

The discriminant of (10) is $64 n$, so that the quadratic factor splits just if $n$ is a perfect square. Put $n=\nu^{2}$ :

$$
T^{2}=S\left(S-(\nu-1)^{3}(\nu+3)\right)\left(S-(\nu+1)^{3}(\nu-3)\right) .
$$

The curve (10) has, in general, a torsion subgroup isomorphic to $\mathbb{Z} / 6 \mathbb{Z}$. The torsion points are $\infty,(0,0)$, two points of inflexion $\left((n-1)^{2}, \pm 4(n-1)^{2}\right)$ of order three, and two points $((n-1)(n-9), \pm 4(n-1)(n-9))$ of order six. 
In the special case when $n=\nu^{2}$ is a square, the torsion subgroup is isomorphic to $\mathbb{Z} / 2 \mathbb{Z} \times \mathbb{Z} / 6 \mathbb{Z}$. There are two additional points $\left((\nu+1)^{3}(\nu \mp 3), 0\right)$ of order two, and four more points of order six, namely $\left(S_{0}, \pm 4 \nu S_{0}\right)$ where $S_{0}=\left(\nu^{2}-1\right)(\nu \pm 1)(\nu \pm 3)$.

To calculate the rank of (6) for particular values of $n$, we follow Birch and Swinnerton-Dyer [1], as exposed by Cassels, Ellison and Pfister [3]: compare also Bremner [2].

Any rational number may be written in the form $\sigma=\delta a^{2} / b^{2}$, where $\delta, a, b$ are integers, $\delta$ is squarefree, $a, b$ are nonnegative, and $a \perp b$ ( $a$ is prime to $b$ ). Substitute in (6) and note that $\tau$ is of shape $\delta a c / b^{3}$, where $c$ is an integer and

$$
\delta c^{2}=\delta^{2} a^{4}+\delta\left(n^{2}-6 n-3\right) a^{2} b^{2}+16 n b^{4},
$$

so that $\delta \mid 16 n b^{4}$. We may assume that $\delta \perp b$. For if $p \mid \delta$ and $p \mid b$, then $p^{2}$ divides the right, and hence the left, of (14): now $\delta$ is squarefree, so $p \mid c^{2}, p^{3}$ divides the left of (14), $p^{3}\left|\delta^{2} a^{4}, p\right| a$, contrary to $a \perp b$.

The existence of a rational point on (6) therefore implies

$$
\delta \mid 2 n \text {. }
$$

[In fact we shall soon see that $n$ odd implies $\delta$ odd, so that $\delta \mid n$.]

If $p_{1}, \ldots, p_{k}$ are the distinct prime factors of $n$, then

$$
\delta= \pm p_{1}^{\varepsilon_{1}} \cdots p_{k}^{\varepsilon_{k}}
$$

with each $\varepsilon_{i}=0$ or 1 , and there are $2^{k+1}$ candidates for $\delta$. From the underlying algebra, it is known that those $\delta$ for which there are solutions form a multiplicative 2-group; however, this is straightforward to verify directly as follows.

Lemma 1. (a) If $\delta(a, b, c)$ is a solution of (14) for $n=n_{1} n_{2}^{2}$ with $n_{1}$ squarefree, and $\delta=\delta_{1} \delta_{2}$ with $n_{1}=\delta_{1} \varepsilon_{1}, n_{2}=\delta_{2} \varepsilon_{2}$, then there is also a solution with $\delta$ replaced by $\varepsilon_{1} \delta_{2}=n / \delta \varepsilon_{2}^{2}$ and $(a, b, c)$ replaced by $\left(4 \varepsilon_{2} b, a, 4 \varepsilon_{2} c\right)$.

(b) If $\delta_{1}\left(a_{1}, b_{1}, c_{1}\right)$ and $\delta_{2}\left(a_{2}, b_{2}, c_{2}\right)$ are solutions of (14) and $\delta_{1}=g d_{1}$, $\delta_{2}=g d_{2}$ with $d_{1} \perp d_{2}$, then $\delta(a, b, c)$ is also a solution, where $\delta=d_{1} d_{2}$, and

$$
a: b=a_{1} b_{1} c_{2}-a_{2} b_{2} c_{1}: d_{1} a_{1}^{2} b_{2}^{2}-d_{2} a_{2}^{2} b_{1}^{2} \text {. }
$$

The corresponding treatment of (10) is to write $S=\Delta A^{2} / B^{2}, T=\Delta A C / B^{3}$ with $\Delta, A, B, C$ integers, $\Delta$ squarefree, $A$ and $B$ nonnegative and $A \perp B$, so that

$$
\begin{aligned}
\Delta C^{2} & =\Delta^{2} A^{4}-2 \Delta\left(n^{2}-6 n-3\right) A^{2} B^{2}+(n-1)^{3}(n-9) B^{4} \\
& =\left[\Delta A^{2}-\left(n^{2}-6 n-3\right) B^{2}\right]^{2}-64 n B^{4} .
\end{aligned}
$$

Note that, from the first form, $\Delta$ divides the squarefree part of $(n-1)(n-9)$, and that if $n>9, \Delta$ is positive (else the left side would be negative and the right side positive). From the second form, $\Delta$ is also positive if $n$ is negative.

Those $\Delta$ for which there are solutions also form a 2-group; the lemma corresponding to Lemma 1 is as follows.

Lemma 2. (a) If $\Delta(A, B, C)$ is a solution of (16) with $(n-1)(n-9)=N_{1} N_{2}^{2}$, $N_{1}$ squarefree, $\Delta=\Delta_{1} \Delta_{2}, N_{1}=\Delta_{1} E_{1}, N_{2}=\Delta_{2} E_{2}$, then

$$
\Delta_{2} E_{1}\left((n-1) E_{2} B, A,(n-1) E_{2} C\right)
$$

is also a solution. 
(b) If $\Delta_{1}\left(A_{1}, B_{1}, C_{1}\right)$ and $\Delta_{2}\left(A_{2}, B_{2}, C_{2}\right)$ are solutions of (16) and $\Delta_{1}=$ $G D_{1}, \Delta_{2}=G D_{2}$ with $D_{1} \perp D_{2}$, then $\Delta(A, B, C)$ is also a solution, where $\Delta=D_{1} D_{2}$ and

$$
A: B=A_{1} B_{1} C_{2}-A_{2} B_{2} C_{1}: D_{1} A_{1}^{2} B_{2}^{2}-D_{2} A_{2}^{2} B_{1}^{2} .
$$

There is always the solution $\delta=1,(a, b, c)=(1,0,1)$, corresponding to the point at infinity on (6), and, if $n=n_{1} n_{2}^{2}$ with $n_{1}$ squarefree, the solution $\delta=n_{1},(a, b, c)=\left(0,1,4 n_{2}\right)$, corresponding to the origin $(0,0)$ on (6). The latter gives a distinct value of $\delta$ just if $n$ is not a perfect square. So the order of the $\delta$-group is at least $2^{1}$, except in the perfect square case, when it may be only $2^{0}$. Then, however, we shall see that the order of the corresponding $\Delta$-group is at least $2^{2}$.

There are always the solutions $\Delta=1,(A, B, C)=(1,0,1)$ and $\Delta=m_{1}$, $(A, B, C)=\left(0,1,(n-1) m_{2}\right)$, where $(n-1)(n-9)=m_{1} m_{2}^{2}$, corresponding to the point at infinity and the origin on the curve (10). So the order of the $\Delta$ group is at least $2^{1}$. When $n=\nu^{2}$ is a perfect square, there are the two further solutions $\Delta=\nu_{1},(A, B, C)=\left((\nu-1) \nu_{2}, 1,0\right)$ and $\Delta=\nu_{3},(A, B, C)=$ $\left((\nu+1) \nu_{4}, 1,0\right)$, where $(\nu-1)(\nu+3)=\nu_{1} \nu_{2}^{2},(\nu+1)(\nu-3)=\nu_{3} \nu_{4}^{2}$. In this instance the order of the $\Delta$-group is at least $2^{2}$.

In all cases, if the orders of the $\delta$ - and $\Delta$-groups are $2^{\gamma}$ and $2^{\Gamma}$, then the rank $r$ is given by

$$
r=\gamma+\Gamma-2 .
$$

\section{LOCAL OBSTRUCTIONS}

The local restrictions on $\delta$ are completely described by Lemmas 3,4 and 5 , which respectively list all exclusions arising from $p=2, p=3$ and $p \geq 5$.

Lemma 3. (a) If $2^{\text {even }} \| n$, i.e., if 2 exactly divides $n$ to an even power, then $\delta \not \equiv 0 \bmod 2$.

If $n$ is odd, write $n=1+2^{\nu} n_{1}$ with $n_{1}$ odd and $\nu \geq 1$.

(b) If $n \equiv 1 \bmod 2$, i.e., $\nu \geq 1$, then $\delta \not \equiv 0 \bmod 2$.

(c) If $n \equiv 1 \bmod 4$, i.e., $\nu \geq 2$, then $\delta \not \equiv 3 \bmod 4$.

(d) If $n \equiv 1 \bmod 8$, i.e., $\nu \geq 3$, and $2^{\text {even }} \|(n-9)$, then $\delta \not \equiv 5 \bmod 8$.

(e) If $n \equiv 1 \bmod 16$, i.e., $\nu \geq 4$, then $\delta \not \equiv 5 \bmod 8$.

Stated positively, in cases (a) and (b) $\delta$ is odd, in case (c) $\delta \equiv 1 \bmod 4$ and in cases $(\mathrm{d})$ and $(\mathrm{e}) \delta \equiv 1 \bmod 8$.

Lemma 4. (a) If $n \equiv 1 \bmod 3$, then $\delta \not \equiv 2 \bmod 3$.

(b) If $3 \| n$, then $\delta \not \equiv 2 \bmod 3$ and $\delta \not \equiv 2 n \bmod 9$.

(c) If $n \equiv 18 \bmod 27$, then $\delta \not \equiv 0 \bmod 3$.

(d) If $n \equiv 9 \bmod 27$, then $\delta \not \equiv 2 \bmod 3$ and $\delta \not \equiv 3 \bmod 9$.

(e) If $n \equiv 36 \bmod 81$, then $\delta \not \equiv 6 \bmod 9$.

(f) If $n \equiv 90 \bmod 243$, then $\delta \not \equiv 6 \bmod 9$.

(g) If $n=3^{\nu} n_{1}, \nu \geq 3$ and $n_{1} \equiv 1 \bmod 3$, then $\delta \not \equiv 2 \bmod 3$ and $\delta \not \equiv 6 \bmod 9$.

Lemma 5. If there is a prime $p \geq 5$ and

(a) $p^{\text {even }} \| n$ and $p \equiv 5 \bmod 6$, then $\delta \not \equiv 0 \bmod p$.

(b) $p \mid(n-1)$, then the Legendre symbol $(\delta \mid p) \neq-1$. 
(c) $p \mid(n-9)$ and $p \equiv 1 \bmod 6$, then $(\delta \mid p) \neq-1$.

(d) $p^{\text {odd }} \|(n-9)$ and $p \equiv 5 \bmod 6$, then $(\delta \mid p) \neq-1$.

The local restrictions on $\Delta$ are completely described by Lemmas 6,7 and 8 , which respectively list all exclusions arising from $p=2, p=3$ and $p \geq 5$.

Lemma 6. (a) If $2 \|(n-1)$, i.e., if 2 exactly divides $n-1$, then $\Delta \not \equiv 0 \bmod 2$.

(b) If $4 \|(n-1)$, then $\Delta \not \equiv 0 \bmod 2$.

(c) If $n \equiv 9 \bmod 32$ and $2^{\text {odd }} \|(n-9)$, then $\Delta \not \equiv 0 \bmod 2$.

(d) If $2 \|(n-1)$, then $\Delta \not \equiv 3 \bmod 4$.

(e) If $2 \mid n$, then $\Delta \not \equiv 3 \bmod 4$.

(f) If $2^{\text {odd }} \| n$, then $\Delta \not \equiv 5 \bmod 8$.

Since $\Delta \mid(n-1)(n-9)$, which is odd if $n$ is even, we may summarize this lemma as follows. If $n$ is even, then $\Delta \equiv 1 \bmod 4$; moreover, if $k \geq 0$ and $n \equiv 2^{2 k+1} \bmod 2^{2 k+2}$, then $\Delta \equiv 1 \bmod 8$. If $n \equiv 3 \bmod 4$, then $\Delta \equiv 1 \bmod 4$; and if $n \equiv 5 \bmod 8$, or if $n \equiv 2^{2 k+5}+9 \bmod 2^{2 k+6}$, then $\Delta$ is odd.

Lemma 7. (a) If $3 \| n$, then $\Delta \not \equiv 2 \bmod 3$.

(b) If $n \equiv 63 \bmod 81$, then $\Delta \not \equiv 2 \bmod 3$.

(c) If $n \equiv 9 \bmod 81$ and $n-9=3^{\nu} n_{1}$ with $n_{1} \equiv 2 \bmod 3$, then $\Delta \not \equiv 2 \bmod 3$.

(d) If $3^{3} \mid n$, then $\Delta \not \equiv 2 \bmod 3$.

(e) If $3 \| n$, then $\Delta \not \equiv n \bmod 9$.

(f) If $n \equiv 18 \bmod 27$, then $\Delta \not \equiv 0 \bmod 3$.

(g) If $n \equiv 63 \bmod 81$, then $\Delta \not \equiv 6 \bmod 9$.

(h) If $n \equiv 9 \bmod 81$ and $n-9=3^{\nu} n_{1}$ with $n_{1} \equiv 2 \bmod 3$, then $\Delta \not \equiv 6 \bmod 9$.

(i) If $3^{3} \mid n$, then $\Delta \not \equiv 3 \bmod 9$.

(j) If $3^{3} \mid n$ and $n=3^{\nu} n_{1}$ with $n_{1} \equiv 2 \bmod 3$, then $\Delta \not \equiv 6 \bmod 9$.

Lemma 8. If there is a prime $p \geq 5$ and

(a) $p \mid n$ and $p \equiv 1 \bmod 6$, then the Legendre symbol $(\Delta \mid p) \neq-1$.

(b) $p^{\text {odd }} \| n$ and $p \equiv 5 \bmod 6$, then $(\Delta \mid p) \neq-1$.

(c) $p^{\text {even }} \|(n-9)$ and $p \equiv 5 \bmod 6$, then $\Delta \not \equiv 0 \bmod p$.

The proofs of the lemmas are omitted. The details present no inherent difficulty and may safely be left to the reader. Alternatively, full details have been written up and are available from the authors if so desired.

\section{DESCENT ARGUMENTS}

For a given value of $n$ and of $\delta$ or $\Delta$, although there is no local obstruction to a global solution of (14) or (16), a fairly extensive search may not reveal a solution. In such cases it is possible to carry out a descent argument, perhaps leading to a proof of impossibility. In any event, this allows a much more efficient search, which in turn may lead to the discovery of a solution.

If the curve (14) is everywhere locally solvable, then so is the associated quadric

$$
c^{2}=\delta \alpha^{2}+\left(n^{2}-6 n-3\right) \alpha \beta+(16 n / \delta) \beta^{2},
$$

and, by the Hasse principle, there is a global solution $a_{0}, b_{0}, c_{0} \in \mathbb{Z}$ with

$$
c_{0}^{2}=\delta a_{0}^{2}+\left(n^{2}-6 n-3\right) a_{0} b_{0}+(16 n / \delta) b_{0}^{2} .
$$


Equations (14) and (19) may be combined and factored to transform the problem into finding integer solutions of the equations

$$
\begin{gathered}
h a^{2}=a_{0} w^{2}-2 c_{0} w v+\left(\delta a_{0}+\left(n^{2}-6 n-3\right) b_{0}\right) v^{2}, \\
h b^{2}=b_{0}\left(w^{2}-\delta v^{2}\right),
\end{gathered}
$$

where $h$ is a squarefree divisor of $(n-1)(n-9) b_{0}$. The corresponding value of $c$ will be given by

$$
h c=c_{0} w^{2}-\left(2 \delta a_{0}+\left(n^{2}-6 n-3\right) b_{0}\right) w v+\delta c_{0} v^{2}
$$

If either (20) or (21) is locally unsolvable for all choices of $h$, then there are no solutions globally, and so no solutions to the original equation.

For a given $h$ it is possible to characterize explicitly those primes $p$ such that $(20),(21)$ are simultaneously locally solvable; but we do not list the relevant conditions here.

If (20), (21) are locally solvable for all primes $p$, then certainly there will be values $h, \beta_{1}, v_{1}, w_{1}$ such that

$$
h \beta_{1}^{2}=b_{0}\left(w_{1}^{2}-\delta v_{1}^{2}\right),
$$

and it can be shown that other solutions of (21) have

$$
v: w=\delta v_{1} p^{2}+2 w_{1} p q+v_{1} q^{2}: \delta w_{1} p^{2}+2 \delta v_{1} p q+w_{1} q^{2}
$$

for integers $p, q$. So search over $p$ and $q$ for ratios $v: w$ that give an integer value for $a$ in (20).

The arguments for the 2-isogenous curve (10) are parallel: the equations corresponding to (19) to (23) being

$$
\begin{gathered}
C_{0}^{2}=\Delta A_{0}^{2}-\left(n^{2}-6 n-3\right)\left(2 A_{0} B_{0}\right)+\frac{(n-1)^{3}(n-9)}{\Delta} B_{0}^{2}, \\
H A^{2}=A_{0} W^{2}-2 C_{0} W V+\left(\Delta A_{0}-2\left(n^{2}-6 n-3\right) B_{0}\right) V^{2} \\
H B^{2}=B_{0}\left(W^{2}-\Delta V^{2}\right), \\
H C=C_{0} W^{2}-\left(\Delta A_{0}-\left(n^{2}-6 n-3\right) B_{0}\right)(2 W V)+\Delta C_{0} V^{2}, \\
H B_{1}^{2}=B_{0}\left(W_{1}^{2}-\Delta V_{1}^{2}\right),
\end{gathered}
$$

and one searches with integers $P$ and $Q$ for ratios

$$
V: W=\Delta V_{1} P^{2}+2 W_{1} P Q+V_{1} Q^{2}: \Delta W_{1} P^{2}+2 \Delta V_{1} P Q+W_{1} Q^{2}
$$

that give an integer value for $A$ in (25).

Example 1. $n=290, \delta=-145$. Equation (19) has a solution $\left(a_{0}, b_{0}, c_{0}\right)=$ $(352,7,13600)$, and $h$ divides $(n-1)(n-9) b_{0}=17^{2} \cdot 281 \cdot 7$. For none of the values $h=1,7,17,119,281,1967,4777,33439$ are the equations (20) and (21) locally solvable, so there are no solutions in this case.

Example 2. $n=682, \delta=-2,\left(a_{0}, b_{0}, c_{0}\right)=(1120,1,2268)$, and $h=$ $458313, p=47, q=1041$ give $v=228930916, w=661668551, a=$ $196340884, b=1088099, c=134423972854224864$, a solution unlikely to be found by direct search. So 682 can be represented by

$$
\begin{aligned}
& x=398726340949893591206=2 \cdot 11 \cdot 13 \cdot 17 \cdot 5563 \cdot 300331 \cdot 49085221, \\
& y=10087757787715867501830=2 \cdot 3^{2} \cdot 5 \cdot 7 \cdot 19 \cdot 631 \cdot 27209489 \cdot 49085221, \\
& z=16043733508910320395=3^{2} \cdot 5 \cdot 7 \cdot 13 \cdot 19 \cdot 41 \cdot 631 \cdot 26539 \cdot 300331,
\end{aligned}
$$


with sum

$10502527862174671413431=11^{2} \cdot 17 \cdot 31 \cdot 41 \cdot 5563 \cdot 26539 \cdot 27209489$ and $1 \mathrm{~cm}$

$$
2 \cdot 3^{2} \cdot 5 \cdot 7 \cdot 11 \cdot 13 \cdot 17 \cdot 19 \cdot 41 \cdot 631 \cdot 5563 \cdot 26539 \cdot 49085221
$$

$.68175137 \cdot 27209489$.

It can, therefore, also be represented by

$$
\begin{aligned}
& \bar{x}=111810428245355640885=3^{2} \cdot 5 \cdot 7 \cdot 19 \cdot 41 \cdot 631 \cdot 26539 \cdot 27209489, \\
& \bar{y}=4419392680958269157=11 \cdot 13 \cdot 19 \cdot 41 \cdot 5563 \cdot 26539 \cdot 300331, \\
& \bar{z}=2778764862277948002778=2 \cdot 11 \cdot 17 \cdot 5563 \cdot 27209489 \cdot 49085221,
\end{aligned}
$$

with sum

$$
2894994683204261912820=2^{2} \cdot 3^{2} \cdot 5 \cdot 7 \cdot 13 \cdot 19 \cdot 631 \cdot 300331 \cdot 49085221 .
$$

Example 3. $n=83, \delta=-1$. The solution $\left(a_{0}, b_{0}, c_{0}\right)=(132,1,908)$ of equation (19) admits the possibilities $h=1,37,41$ and 1517 in equations (20), (21), each of which leads to a locally solvable pair of quadrics. However, an extended search failed to find any rational points for any of the values of $h$. Computation of the $L$-series $L_{E}(s)$ associated with the elliptic curve $E$ of this example shows that $L_{E}(1) \neq 0$ whence the conjectures of Birch and Swinnerton-Dyer imply that the rank of $E$ is zero (actually, given results of Kolyvagin, all we need is that our curve be modular), so that there are no nontrivial rational solutions of ( 1 ) for $n=83$. We undertook a further descent on each pair of equations corresponding to the four values of $h$, involving a considerable amount of numerical detail. The arguments do indeed show that for none of the values of $h$ do the curves $(20),(21)$ possess rational points. Thus there are no solutions of (14) for $n=83, \delta=-1$. It then follows (cf. Lemma 1) that (14) does not have solutions for $n=83, \delta=-83$. It remains to discuss $\delta=37$ or 41 , but we have not carried out a further descent in these instances.

Example 4. $n=212, \Delta=29 .\left(A_{0}, B_{0}, C_{0}\right)=(1329,1,952), H=-53$,

$$
\begin{gathered}
(P, Q)=(223,983), \quad(W, V)=(6875172,1286777), \\
(A, B, C)=(25632749,118963,3423494950408888), \\
x=-108330247204631183866777136086499984804751255, \\
y=226158369568634413878822342329578954739317052, \\
z=556924334185535574920737272977470281885268, \\
x+y+z=3 \cdot 5 \cdot 7 \cdot 11 \cdot 13 \cdot 17 \cdot 37 \cdot 41 \cdot 53 \cdot 61 \cdot 277 \cdot 643 \cdot 2833 \\
\cdot 9151 \cdot 21193 \cdot 13376239 \cdot 72243533 .
\end{gathered}
$$

As we have seen, solutions obtained via values of $\Delta$ cannot have all of $x, y$ and $z$ positive.

\section{RESUlTS}

By computation on the curves (6) and (13) we searched for solutions of (1) over the range $-1000 \leq n \leq 1000$. In all cases the methods of $\S 5$ give an upper 


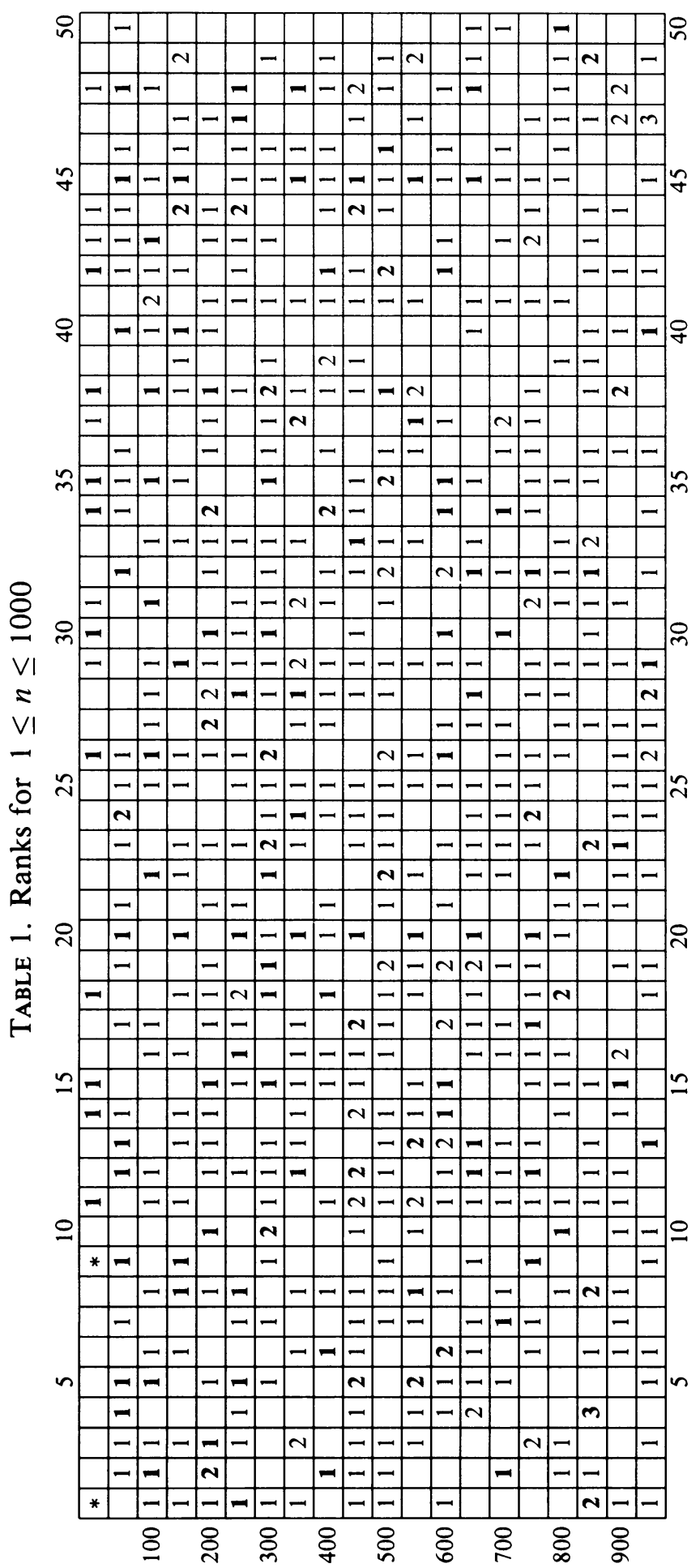




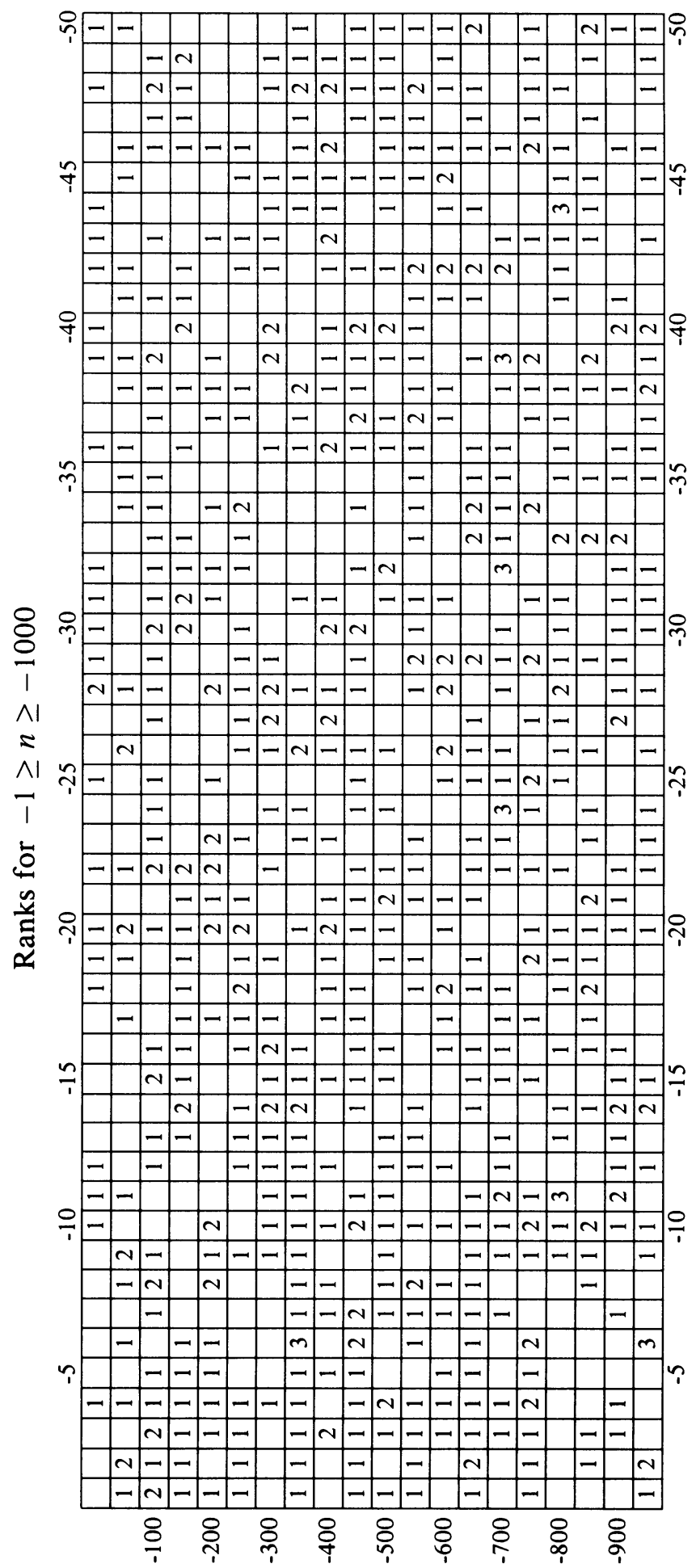


bound for the rank, and in many cases allow its explicit determination. In any event, the Selmer conjecture predicts the rank parity. As a check, and in order to obtain the value of the rank with reasonable assurance, we assumed that our elliptic curves are modular, i.e., that they satisfy the Taniyama-Weil conjecture, and worked with their corresponding $L$-series, $L_{E}(s)$. Taking on faith the conjectures of Birch and Swinnerton-Dyer, then the evaluation of $L_{E}(1)$ and $L_{E}^{\prime}(1)$ together with the explicit numerical solutions found by computer search allowed us to deduce the exact value of the rank within the range under consideration. PARI-GP was used for this computation; for details of the underlying theory and series summation, see, e.g., Zagier and Kramarz [6].

Results are given in Table 1, where each entry is the rank (modulo the conjectures of Birch and Swinnerton-Dyer). An asterisk denotes a singular value of $n$ and a blank denotes rank zero. Boldface type is used for those $n$ for which there is a positive representation. Note that there exist representations for the singular values of $n$, and also for $n=10$, despite its curve being of rank zero.

We have compiled a table of solutions $(x, y, z)$ of (1). For each $n$ we give $r$ independent solutions (where $r$ is the rank of the curve), except in about $5 \%$ of the cases, where solutions have still to be found. We confidently expect that this table gives the "smallest" possible solutions in each instance. The measure of the size of a point on an elliptic curve is given by its height, and our search process finds solutions in increasing order of height. In rank-one cases the conjectures of Birch and Swinnerton-Dyer allow us to estimate

height of a generator $\times$ the order of $w$,

where $w$ is the Tate-Shafarevich group. Since the search process finds all solutions of small height, we can confidently predict the (perfect square) order of $w$

TABLE 2. Solutions for positive rank in the range $1 \leq n \leq 100$

\begin{tabular}{rrrrrrrr}
\hline$n$ & $x$ & $y$ & $z$ & $n$ & $x$ & $y$ & $z$ \\
\hline 11 & 1 & 2 & 3 & 63 & 5 & 119 & 170 \\
14 & 2 & 3 & 10 & 64 & 1 & -40 & 104 \\
15 & 1 & 2 & 6 & 67 & 17 & -105 & 1428 \\
18 & 2 & 3 & 15 & 69 & 5989 & -747565 & 1155990 \\
26 & 1 & 6 & 14 & 70 & 70 & 551 & 3654 \\
29 & 5 & -26 & 195 & 71 & 11 & -116 & 957 \\
30 & 10 & 77 & 165 & 73 & 4 & -19 & 380 \\
31 & 3 & -11 & 132 & 74 & 5 & 22 & 270 \\
34 & 1 & 6 & 21 & & 287 & -32480 & 53505 \\
35 & 3 & 10 & 65 & 75 & 10153 & -58656 & 957719 \\
37 & 7 & -22 & 385 & 76 & 18 & -238 & 1683 \\
38 & 90 & 391 & 2210 & 82 & 119 & 475 & 7106 \\
42 & 561 & 6450 & 13889 & 84 & 2 & -78 & 247 \\
43 & 39 & -984 & 2665 & 85 & 23 & -95 & 2622 \\
44 & 777 & -40810 & 74518 & 86 & 21 & -37 & 4144 \\
48 & 70633 & -3329130 & 6685382 & 90 & 1386 & 35226 & 81473 \\
52 & 29 & -45 & 4176 & 92 & 203 & -8778 & 27550 \\
53 & 85 & -141 & 11186 & 93 & 124285 & -32337349 & 43783194 \\
54 & 55 & 595 & 2002 & 94 & 1005720 & -44186077 & 139210005 \\
55 & 1 & 14 & 35 & 95 & 138 & 357 & 8855 \\
57 & 14 & -174 & 1015 & 96 & 11289 & -611168 & 1696583 \\
59 & 2 & 15 & 85 & 98 & 1221 & 2950 & 79550 \\
62 & 5075 & 128050 & 160602 & 100 & 5 & -56 & 595 \\
\hline
\end{tabular}


by calculating the height of our known solution $P$; this provides a check that $P$ is a generator. For example, if $n=-568$, then the height of a generator $\times$ the order of $\omega \approx 115.2$. If $|w| \geq 9$, then a generator would have height at most 12.8 and our search tells us that there are no such points. Since the height of our known solution $P$ is $\approx 28.8$, it follows that $|w|=4$ and $P$ is a generator. We have also checked one or two examples of rank two (necessitating the evaluation of $\left.L_{E}^{\prime \prime}(1)\right)$ to verify that we have found a system of generators.

The full table with solutions over the range $-1000 \leq n \leq 1000$ is available on request.

The following are rank statistics for $-1000 \leq n \leq 1000$ :

$\begin{array}{cccccc}\text { Range } & \text { rank } 0 & \text { rank 1 } & \text { rank 2 } & \text { rank 3 } & \text { Total } \\ -1000 \leq n<0 & 416 & 476 & 101 & 7 & 1000 \\ 0<n \leq 1000 & 440 & 494 & 62 & 2 & 998 \\ \text { Total } & 856(42.8 \%) & 970(48.5 \%) & 163(8.2 \%) & 9(0.5 \%) & 1998\end{array}$

Negative values of $n$ appear to give slightly higher ranks than positive ones do, but this may be due to the existence of sequences of parametric solutions, which, although of asymptotic density zero, account for 86 cases of positive rank in the interval $-1000 \leq n<0$. Indeed, five of the seven rank-3 examples in this interval $(n=-356,-724,-732,-844$ and -956$)$ are included in the following examples:

(a) $n=-\left(k^{2}-5\right) \quad(k \geq 3)$ :

$$
(x, y, z)=\left(k+1, k-1,-k\left(k^{2}-1\right) / 2\right) .
$$

(b) $n=-\left(k^{2}+3\right) \quad(k \geq 3)$ :

$(x, y, z)=\left(\left(k^{3}+3 k^{2}+4 k+4\right) / 2,\left(k^{3}-3 k^{2}+4 k-4\right) / 2,-k\left(k^{4}+3 k^{2}+4\right) / 4\right)$.

(c) $n=-(k-1)(k+2) \quad(k \geq 3)$ :

$$
(x, y, z)=(1, k,-k(k+1)) .
$$

Pursuit of these sequences has revealed a rank-4 example, $n=-9796$, for which a set of independent solutions is afforded by $(\delta, a, b)=(2449,106,1)$ and $(\Delta, A, B)=(9805,97,1),(190217,14,1),(990305,12,1)$.

There are numerous other parametric solutions, though mostly of exponential type. Perhaps the most pleasing are

(d) $n=v_{4 k}+8$ :

$$
(x, y, z)=\left(u_{2 k-1}, u_{2 k+1}, u_{2 k-1} v_{2 k} u_{2 k+1}\right) .
$$

(e) $n=u_{2 k}^{2}$ :

$$
(x, y, z)=\left(-u_{2 k-1}, u_{2 k+1}, u_{2 k-1} u_{2 k} u_{2 k+1}\right) \text {, }
$$

where $u_{k}, v_{k}$ are the Fibonacci and Lucas numbers, satisfying $w_{k+1}=w_{k}+$ $w_{k-1}, u_{1}=u_{2}=v_{1}=1, v_{2}=3$.

\section{CONCLUSION}

As a comment on the appositeness of Melvyn Knight's problem, we glance briefly at the corresponding problems with two or four variables. If

$$
(x+y)\left(\frac{1}{x}+\frac{1}{y}\right)=n,
$$


then $x^{2}+(2-n) x y+y^{2}=0$ has an integer solution only if $(2-n)^{2}-4$ is a square, i.e., only if $n=0$ or 4 (when $x=\mp y$ ). If

$$
(x+y+z+w)\left(\frac{1}{x}+\frac{1}{y}+\frac{1}{z}+\frac{1}{w}\right)=n,
$$

then there are infinitely many representations of every $n$, e.g.,

$$
(x, y, z, w)=\left(m^{2}+m+1, m(m+1)(n-1),(m+1)(n-1),-m(n-1)\right)
$$

for any integer $m$. The least $n$ with a positive representation is 16 , and it seems likely that for $n \geq 16$ there is always such a representation, e.g., $16(1,1,1,1) ; 17(2,3,3,4) ; 18(1,1,2,2) ; 19(8,9,18,21) ; 20(1,3,3,3)$.

\section{ACKNOWLEDGMENTS}

We are grateful to Andy Guy for largely automating the descent process with efficient $\mathrm{C}++$ programs, and to Graham Birtwistle and Brian Wyvill for arranging for considerable CPU time in the Computer Science Department at The University of Calgary. Research was assisted by a grant from the Natural Sciences \& Engineering Research Council of Canada.

\section{BIBLIOGRAPHY}

1. B. J. Birch and H. P. F. Swinnerton-Dyer, Notes on elliptic curves. II, J. Reine Angew. Math. 218 (1965), 79-108.

2. Andrew Bremner, A geometric approach to equal sums of sixth powers, Proc. London Math. Soc. (3) 43 (1981), 544-581.

3. J. W. S. Cassels, W. J. Ellison, and A. Pfister, On sums of squares and on elliptic curves over function fields, J. Number Theory 3 (1971), 125-149.

4. L. J. Mordell, Diophantine equations, Academic Press, New York, 1969.

5. J. H. Silverman, The arithmetic of elliptic curves, Springer-Verlag, New York, 1986.

6. D. Zagier and G. Kramarz, Numerical investigation related to the $L$-series of certain elliptic curves, J. Indian Math. Soc. 52 (1987), 51-69.

Department of Mathematics, Arizona State University, Tempe, Arizona 85287-1804

E-mail address: abremner@math.la.asu.edu

Department of Mathematics and Statistics, University of Calgary, Calgary, Alberta, Canada T2N 1N4

E-mail address: rkg@cpsc.ucalgary.ca

Department of Mathematics, Statistics and Computing Science, Dalhousie UniverSity, Halifax, Nova Scotia, Canada B3H 3J5

E-mail address: rjn@cs.dal.ca 